\title{
Geo-visualization of antibiotic resistance based on web map service
}

\author{
Taekeon Hwang $^{\mathrm{a}, *}$, Kangmin Kim ${ }^{\mathrm{b}}$, Chul-Sue Hwang ${ }^{\mathrm{c}}$, Chang-Jun Cha ${ }^{\mathrm{d}}$ \\ ${ }^{a}$ Department of Geography, Kyung Hee University, htkgeo@khu.ac.kr \\ ${ }^{b}$ Department of Geography, Kyung Hee University, kingkangzza@gmail.com \\ ${ }^{c}$ Department of Geography, Kyung Hee University, hcs@khu.ac.kr \\ ${ }^{d}$ Department of Systems Biotechnology, Chung-Ang University, cjcha@cau.ac.kr \\ * Corresponding author
}

Keywords: Antibiotic resistance, Geo-visualization, WMS, WebGIS

\section{Abstract:}

An antibiotic resistance is a serious problem on health and humanity. Our human society needs to understand the threat of antibiotic resistance. To geo-visualize the location, biotic information and spatial pattern of antibiotic resistance matters is an effective way for intuitive findings. We focused on the antibiotic resistance issue of Han-river in South Korea and analyzed their spread, as well as range of influence.

We developed the geo-database of antibiotic resistance matters and represented spatial information based on WMS (Web Map Service) which is adjusted to OGC (Open Geospatial Consortium) specifications. We designed correlated data schema structure of antibiotic matters, degrees of resistance, water quality and location of the sampled points on the river. Our WMS system followed the open source architecture and Q-GIS to make geo-server. This system was organized server-client structure.

As for antibiotic resistance map on our WMS system, we illustrated the infographics and related tables of many kinds of information about antibiotic resistance. The WMS system represented simple spatial information, temporal variation of antibiotic resistance and degrees of correlation between antibiotic resistance and human activities such as emissions of antibiotic resistance matters on hospital or pharmacy. We also considered domestic sewage discharged into river through sewage processing facilities.

This system will be contributed to reducing social cost and economic loss caused by antibiotic resistance spreads. These web maps will provide users with correct information on threat of antibiotic resistance. 